\title{
DECIDING THE NATURE OF THE COARSE EQUATION THROUGH MICROSCOPIC SIMULATIONS: THE BABY-BATHWATER SCHEME*
}

\author{
JU LI ${ }^{\dagger}$, PANAYOTIS G. KEVREKIDIS ${ }^{\ddagger}$, C. WILLIAM GEAR ${ }^{\S}$, AND \\ IOANNIS G. KEVREKIDIS 『
}

\begin{abstract}
Recent developments in multiscale computation allow the solution of coarse equations for the expected macroscopic behavior of microscopically evolving particles without ever obtaining these coarse equations in closed form. The closure is obtained on demand through appropriately initialized bursts of microscopic simulation. The effective coupling of microscopic simulators with macrosocopic behavior requires certain decisions about the nature of the unavailable coarse equation. Such decisions include (a) the highest spatial derivative active in the coarse equation, (b) whether the equation satisfies certain conservation laws, and (c) whether the coarse dynamics is Hamiltonian. These decisions affect the number and type of boundary conditions as well as the algorithms employed in good solution practice. In the absence of an explicit formula for the temporal derivative, we propose, implement, and validate a simple scheme for deciding these and other similar questions about the coarse equation using only the microscopic simulator. Simulations under periodic boundary conditions are carried out for appropriately chosen families of random initial conditions; evaluating the sample variance of certain statistics over the simulation ensemble allows us to infer the highest order of spatial derivatives active in the coarse equation. In the same spirit we show how to determine whether a certain coarse conservation law exists or not, and we discuss plausibility tests for the existence of a coarse Hamiltonian or integrability. We believe that such schemes constitute an important part of the equation-free approach to multiscale computation.
\end{abstract}

Key words. equation-free, multiscale computation, identification, microscopic simulator, coarse dynamics

AMS subject classifications. 65N99, 65C35, 82C21, 93E12

DOI. $10.1137 / \mathrm{S} 1540345902419161$

1. Introduction. It is often the case that a microscopic or fine description of a physical system is available, while we are interested in its macroscopic or coarse behavior. Consider, as an example, a biased random walk model for which the particle density asymptotically evolves according to a macroscopic law such as the Burgers equation. Typically, the study of macroscopic behavior starts with obtaining a closed PDE-level description (a coarse equation) for the time evolution of the expected or ensemble-averaged fields of a few, low order moments of the microstate phase-space distribution. For our example, this would be the 0th moment, the density field. Then an array of mathematical and computational tools (numerical integration, fixed-point algorithms, etc.) can be brought to bear on the coarse equation.

Over the last few years, we have been developing a class of numerical algorithms

*Received by the editors December 7, 2002; accepted for publication (in revised form) May 13, 2003; published electronically July 17, 2003. This work was partially supported by Honda R\&D Co., Ltd. and the OSU Transportation Research Endowment Program (JL), the National Science Foundation (IGK, PGK), AFOSR (CWG, IGK) and the Clay Institute (PGK).

http://www.siam.org/journals/mms/1-3/41916.html

${ }^{\dagger}$ Department of Materials Science and Engineering, Ohio State University, Columbus, OH 43210 (lij@mse.eng.ohio-state.edu).

${ }^{\ddagger}$ Department of Mathematics and Statistics, University of Massachusetts, Amherst, MA 010034515 (kevrekidis@math.umass.edu).

$\S$ NEC Research Institute, 4 Independence Way, Princeton, NJ 08540 (cwg@nec-labs.com).

IDepartment of Chemical Engineering and PACM, Princeton University, Princeton, NJ 08544 (yannis@princeton.edu). 
which attempt to analyze the coarse behavior without ever obtaining the coarse equation in closed form $[1,2,3]$. The common character of these schemes is to use short, appropriately initialized bursts of microscopic simulations to estimate the quantities which, if the coarse equation were available, we would simply evaluate using the equation itself. Such quantities, estimated on demand, include the time derivative of the evolving coarse fields, to be used in coarse projective integration [4], or the effect of the time-evolution operator for the implicit coarse Jacobian, to be used in NewtonKrylov-type contraction mappings like the recursive projection method (RPM) [5, 6] or in eigenvalue/vector computations. These methods are based on matrix-free large scale scientific computing, and we collectively refer to them as equation-free methods. What makes these computations possible is the assumption of a separation of time scales or large spectral relative gaps in the collective dynamics. When a coarse description is conceptually possible, one typically finds that the hierarchy of coupled equations involving higher cumulants of the microscopic distribution constitutes a singularly perturbed problem: higher-order cumulants become, in the course of microscopic simulation, quickly slaved to (become deterministic functionals of) the lower-order cumulants. The consequence of slaving, realized in the computer as a black box, embodies the closure that allows us to solve for the coarse behavior. Fundamentally it is no different than if the closures are expressed in closed form first and then evaluated later. This way of thinking and newly developed computing technology can in practice exceed the traditional approach in both accuracy and cost, especially if the constitutive relation is multidimensional and nonlinear. An example of this type is given in $[7,8]$. An additional advantage of such methods is their ability to detect parametric regimes where the present closure model is inadequate, and hence appropriate refinements (including higher-order moments) are necessary.

In the projective integration method [4] one takes advantage of the slow dynamics of the coarse variables to carry out only bursts of microscopic simulations connected via extrapolations and/or interpolations over gaps of time. In the same spirit, but now in space, we have developed the so-called gaptooth scheme $[9,10]$ by evolving the system only in an array of small spatial boxes (the teeth) separated by empty regions (the gaps). Clearly, the two methods are closely related by the physics of the problem, i.e., the dispersion relation of the coarse excitations. Indeed we can have a combined gaptooth-projective integration scheme, and in fact the spatial and temporal gaps then need to be chosen properly to satisfy exactly the same Courant-Friedrichs-Levy $(\mathrm{CFL})$ condition as in a finite-difference scheme; this is the focus of another paper [1]. Here, we simply want to point out the fact that in the gaptooth method, the teeth communicate with each other via appropriate boundary conditions for the microscopic simulations performed inside them. And here lies the raison d'être of this paper.

It is well known that certain features of a given equation affect the nature of the appropriate numerical solver. It might be appropriate, for example, to integrate a Hamiltonian dynamical system using a symplectic integrator; often, finite difference solvers of PDEs are built to preserve certain properties of the PDE, such as conservation laws. Most importantly, the highest spatial order of an evolution equation critically affects the types of boundary conditions leading to a well-posed problem.

In a completely analogous manner, the way in which the microscopic model is solved separately in each tooth in the gaptooth scheme, and the boundary conditions applied to the edges of each tooth, must respect the nature of the unavailable equations and their order. Furthermore, gaptooth algorithms compatible with conservation laws (e.g., using fluxes to estimate temporal derivatives; see, for example, [11]) are 
predicated upon knowing that the unavailable equation possesses certain conservation laws.

When the closed-form equation is available, some of these questions (e.g., the order of the highest spatial derivative in an evolution equation) can be answered by direct inspection. Other issues (such as the existence of conservation laws or integrability) may, in the case of closed-form equations, be relatively obvious or may require a lot of work.

What we explore in this paper is the development of computer-assisted methodologies to answer the above questions when closed-form equations are not available. The idea is that we can probe the consequences of these answers on the dynamics of the unavailable coarse equations using microscopic particle- or agent-based simulators by trying out large classes of appropriately chosen initial conditions. We will illustrate what we call the baby-bathwater algorithm on examples of particle systems realizing the Burgers and Korteweg-de Vries (KdV) equations, for the task of inferring the highest order of spatial derivative on the right-hand side, and for answering questions concerning coarse conservation laws.

The paper is organized as follows. In section 2 we briefly present our illustrative particle-based example. In section 3 we discuss the determination of the highest order spatial derivative active in the unavailable equation. In section 4 we explore the possible existence of conservation laws. In the concluding section we discuss the scope and limitations of the procedure, as well as additional questions that may be addressed through this approach. An interesting twist about reverse coarse integration arises in discussing the exploration of possible coarse Hamiltonianity of the unavailable coarse equation.

2. Numerical experiment setup. Our illustrative example will be based on simple numerical experiments. We will first, as a sanity check, demonstrate the approach using a traditional numerical simulator of a known evolution equation as a black box. We will then substitute the simulator of the known continuum equation with a particle-based simulator and repeat the procedure. Our first illustration will be the Burgers equation,

$$
u_{t}+u u_{x}=\nu u_{x x}
$$

as well as a particle-based simulator constructed so that the evolution of its density resembles the Burgers evolution. Since one of the issues to be explored is the number and type of boundary conditions in evolving the equation, our simulations must be possible without this a priori knowledge. We therefore use periodic boundary conditions (PBCs) enforced on $x \in[0,2 \pi)$ in all our exploratory simulations. One of the attractive features of the Burgers equation is that for any initial profile $u(x, t=0)$, and even with PBCs, the Cole-Hopf transformation $[12,13]$ provides an analytical solution. The accuracy of the numerics can thus be checked directly.

A biased random walker-based particle simulator mimicking the Burgers dynamics is constructed, following the proof of strong convergence of such methods [14]. As a reference, the diffusion equation,

$$
u_{t}=\nu u_{x x}
$$

has the well-known microscopic realizations of Langevin dynamics or unbiased random walkers. It is not too difficult to conjure up a similar system: a unit mass $\int u(x, t) d x=$ 1 in the coarse description corresponds to $Z$ walkers, where $Z$ is a large integer 
constant. In the simulation, $N$ random walkers move on $[0,2 \pi)$ at discrete time-steps $t_{n}=n h$. At each step, (a) the walkers' positions $\left\{x_{i}\right\}$ are sorted, and (b) each walker $i$ checks out the position of the walker $m$-places ahead, $x_{i}^{m+}$, and $m$-places behind, $x_{i}^{m-}$ (properly accounting for PBCs, of course). The difference $x_{i}^{m+}-x_{i}^{m-}$ is inversely proportional to the local density of walkers; therefore (c) every walker moves by $\Delta x_{i}$ sampled from $N\left(m h / Z\left(x_{i}^{m+}-x_{i}^{m-}\right), 2 \nu h\right)$, a biased Gaussian distribution. The $x_{i}$ 's are then wrapped around to $[0,2 \pi)$, and the process repeats. This achieves a coarsegrained flux of $j \equiv u^{2} / 2-\nu u_{x}$ as motivated by (2.1) by assigning each walker a drift speed of $u / 2$. Here, $m$ is analogous to the number of directly interacting neighbors in a molecular dynamics simulation. A detailed study of the features of this particle model, including microscopic pair correlations, is reported elsewhere [15]; it is not, however, an important issue for this paper. It is only for benchmarking purposes that the relation to a known macroscopic equation is brought up. One can start by presenting a microscopic evolution law, without knowing anything about its corresponding coarse equation, and apply our algorithms directly.

Relating the fine with the coarse description requires the use of lifting and restriction operators $\hat{\mu}, \hat{\mathcal{M}}[6]$. Lifting $\hat{\mu}$ constructs a particle system sample (a phase point) conditioned on some of the phase-space distribution's lower moment fields (here the zeroth moment, the coarse density field); it clearly is a one-many operator, and multiple microscopic realizations of a given macroscopic initial condition are often required $[1,6,16]$. The restriction operator $\hat{\mathcal{M}}$-here estimating the moment fields of a given particle distribution [17] — is a form of projection. Clearly, $\hat{\mathcal{M}} \hat{\mu}$ should be the identity, or close to it, due to noise effects. The lifting and restriction operators we construct for this work, with $u$ interpreted as the coarse density on $[0,2 \pi)$ with PBCs, are given in Appendix A. Figure 1 shows a result of the "reversibility test": we randomly generate a coarse density $u(x)$, lift it to a random walker sample, then restrict back to $\tilde{u}(x)$, and observe the very good agreement between $\tilde{u}(x)$ and $u(x)$. Notice that the only point where this agreement may be less satisfactory is close to local maxima or minima, where the derivative changes sign.

While the proximity of our particle scheme to the Burgers evolution is not the issue in this paper, we briefly illustrate the correspondence of the evolution of an initial profile through the two approaches. Figure 2 shows the analytical solution obtained through the Cole-Hopf transformation; it also contains the result of a 21991particle simulation after the configurations have been processed by the $\hat{\mathcal{M}}$ operator of Appendix A to extract the coarse density field. A small value of viscosity $\nu=0.1$ is picked to accentuate the behavior of the steepening wavefront with time. The microscopic simulation clearly captures the important features of the coarse behavior. Ensemble averaging with the same initial condition in coarse field $u(x, 0)$ would reduce the error, but, as we can see, even a single microscopic simulation using a reasonable number of particles may still perform quite well.

Last, we mention the existence of particle methods $[14,18,19,20]$ in solving PDEs such as the following KdV equation:

$$
u_{t}=6 u u_{x}-u_{x x x}
$$

which can be formulated as conservation laws. In this paper we use our construction above for the Burgers example. We should highlight once more that our ultimate goal is not to construct particle solvers of given equations but rather to deduce features of the unavailable coarse equations for given microscopic schemes. 


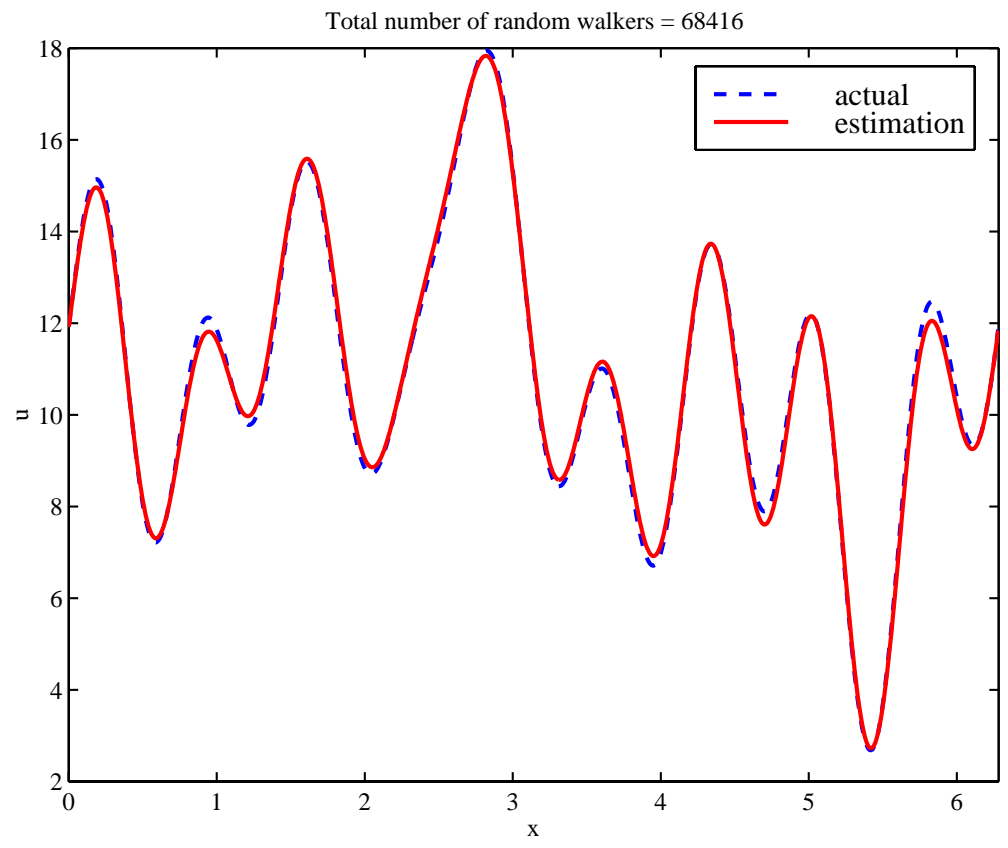

Fig. 1. Reversibility test $(\hat{\mathcal{M}} \hat{\mu} \approx \hat{I})$ of the $\hat{\mu}, \hat{\mathcal{M}}$ operators constructed in Appendix $A: Z=1000$, $M=10$, and $u(x)$ is generated by randomly drawing $n a_{n}, n b_{n}$ from $N(0,1), n=1, \ldots, M$ (see the appendix for details).

3. Identifying the highest spatial order of coarse variables. As we mentioned in the introduction, system identification lies at the heart of the equation-free approach. Here, we suppose the coarse dynamics follows a certain time-evolution equation of the following form:

$$
u_{t}=f\left(u, u_{x}, u_{x x}, \ldots, u_{x}^{(N)}\right),
$$

which is unavailable. However, suppose we have already identified $u$, the coarse variable for which we believe a coarse deterministic equation exists, and constructed the lifting and restriction operators that connect macro-/micro-descriptions. We seek a general approach to answer qualitative questions such as (a) what $N$ is and (b) whether $f$ can be written as $-\nabla \cdot \mathbf{j}$, without having $f$ in closed form. The motivation for this is that equation-free computation (for example through the gaptooth scheme) does not require knowledge of $f$, but is affected by the knowledge of $N$, through teeth boundary conditions. What we have is a microscopic simulator embodied in a computer code that can be initialized at will, which we use as a coarse input/output (I/O) black box. By probing the I/O response of the black box, the question is whether we can decide on (a) and/or (b).

It may appear that we are trying to answer a circular question: in order to probe the coarse I/O response of a microscopic simulator we need to run it, and to run it we need well-posed boundary conditions which, among other factors, depend on (a) and (b). To cut the knot, we use the Born-von Karman PBCs at the decision stage. We are going to assume that the microscopic simulations can be carried out in PBCs, which is an option prevalent among microscopic simulators. This enables us to probe the system's response to only the initial profile $u(x, 0)$ input. 


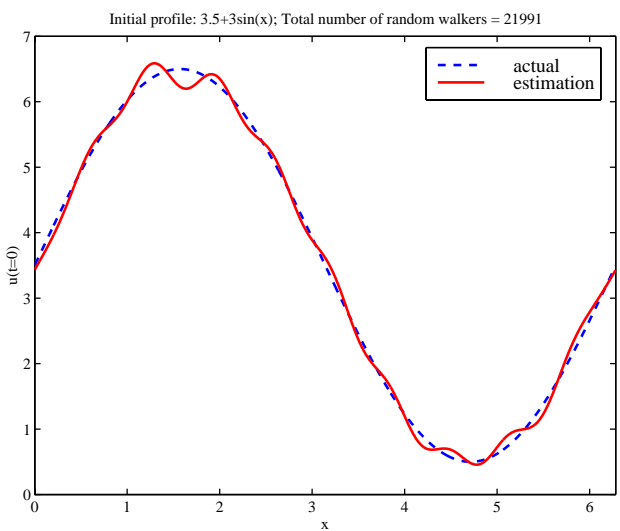

(a)

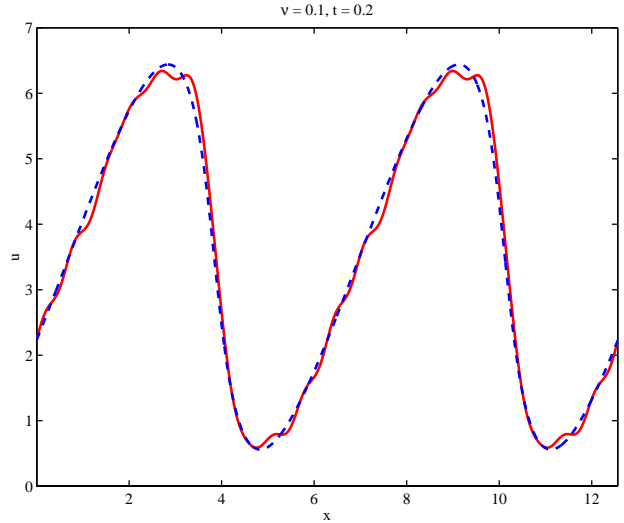

(b)

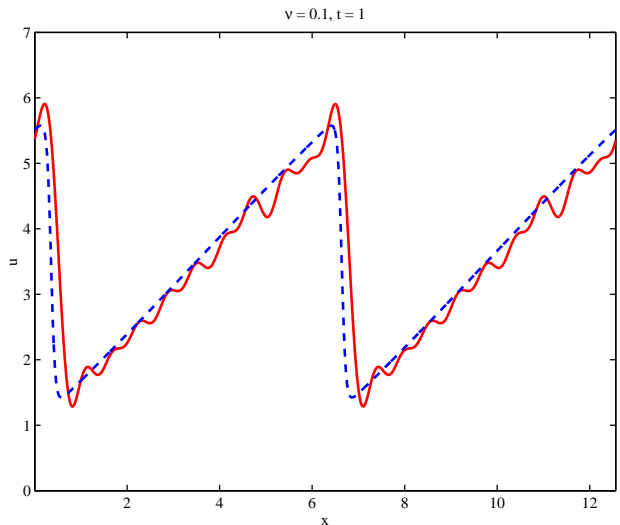

(c)

FIG. 2. Analytical solution of the Burgers equation at $\nu=0.1$ and solutions of our particlebased scheme. (a) Initial condition $u(x, 0)=3.5+3 \sin (x)$; comparison between designated profile and after $\hat{\mathcal{M}} \hat{\mu}$ for $Z=1000, M=10$; (b) comparison of analytical solution and simulation (after restriction) at $t=0.2$ (tiled for ease of seeing the wave steepening); and (c) $t=1$. The microscopic simulation is carried out with $m=10, h=5 \times 10^{-4}$. 
The so-called baby-bathwater identification scheme works as follows:

(i) Take an integer $n$, starting from 1.

(ii) Pick a random point $x_{0}$ in the spatial periodic box.

(iii) Generate $n$ random numbers, designated as $u\left(x_{0}, 0\right), u_{x}\left(x_{0}, 0\right), u_{x x}\left(x_{0}, 0\right)$, $\ldots, u_{x}^{(n-1)}\left(x_{0}, 0\right)$ of $u(x, 0)$.

(iv) Generate a conditionally random profile $u(x, 0)$ compatible with the $\mathrm{PBC}$ and consistent with the above $u\left(x_{0}, 0\right), u_{x}\left(x_{0}, 0\right), u_{x x}\left(x_{0}, 0\right), \ldots, u_{x}^{(n-1)}\left(x_{0}, 0\right)$ requirements. This can almost always be accomplished, for example, by summing $2 L$ sine and cosine harmonics of the PBC:

$$
u(x, 0)=b_{0}+\sum_{i=1}^{L} a_{l} \sin (l x)+b_{l} \cos (l x), \quad x \in[0,2 \pi),
$$

with $L>\lceil n / 2\rceil$. Because we have $2 L+1$ coefficients, even though there are $n$ constraints to satisfy, we still have some random degrees of freedom left in (3.2). In practice, this initialization can be accomplished by applying conjugate gradient minimization of the $n$-dimensional residual norm starting from a random $\left\{a_{l}, b_{l}\right\}$ vector.

(v) Lift $u(x, 0)$ of $(3.2)$, run it in the microscopic simulator for time $\Delta$, restrict it back to $\tilde{u}(x, \Delta)$, and estimate:

$$
\tilde{u}_{t}\left(x_{0}, 0\right) \equiv \frac{\tilde{u}\left(x_{0}, \Delta\right)-\tilde{u}\left(x_{0}, 0\right)}{\Delta} .
$$

Note that $\tilde{u}\left(x_{0}, 0\right)$ is used here instead of $u\left(x_{0}, 0\right)$ in the finite difference. This will cancel out some numerical noise from the lifting and restriction operations.

(vi) Repeat step (v) $I$ times to obtain an ensemble-averaged $\tilde{u}_{t}\left(x_{0}, 0\right)$ to reduce the microscopic noise.

(vii) Repeat step (iv) $J$ times; collect the $\tilde{u}_{t}\left(x_{0}, 0\right)$ estimates:

$$
\left(\tilde{u}_{t}^{1}\left(x_{0}, 0\right), \tilde{u}_{t}^{2}\left(x_{0}, 0\right), \ldots, \tilde{u}_{t}^{J}\left(x_{0}, 0\right)\right) ;
$$

compute the sample variance $\sigma^{2}\left(\tilde{u}_{t}\left(x_{0}, 0\right)\right)$.

(viii) Repeat step (ii) $K$ times; compute the averaged sample variance $\left\langle\sigma^{2}\left(\tilde{u}_{t}\right)\right\rangle_{n}$.

(ix) Go back to step (i); $n \rightarrow n+1$. $N$ is identified when from $n=N$ to $n=N+1$ the averaged sample variance $\left\langle\sigma^{2}\left(\tilde{u}_{t}\right)\right\rangle_{N+1}$ decreases drastically to practically 0 .

Figure 3 shows such families of constructed initial profiles with progressively more controlled initial derivatives. The basic idea is very simple: even though $f$ could have complicated functional dependencies on $u, u_{x}, u_{x x}, \ldots, u_{x}^{(N)}$ if they are all fixed, $u_{t}$ should have no dispersion even as $u_{x}^{(N+1)}, u_{x}^{(N+2)}, \ldots$ are varied randomly. The "critical integer order" $N$ is identified when the variance at $N$ controlled derivatives $u\left(x_{0}, 0\right), u_{x}\left(x_{0}, 0\right), u_{x x}\left(x_{0}, 0\right), \ldots, u_{x}^{(N-1)}\left(x_{0}, 0\right)$ jumps to a finite value; we then have already thrown out the "baby" (the highest relevant spatial derivative $u_{x}^{(N)}$ ) with the "bathwater" (the higher, nonrelevant ones).

It is important to recognize that the time derivative estimation (3.3) does not occur instantaneously. A short "healing" period should elapse, during which the higher cumulants of the lifted phase space distribution become functionals of the lower order, 

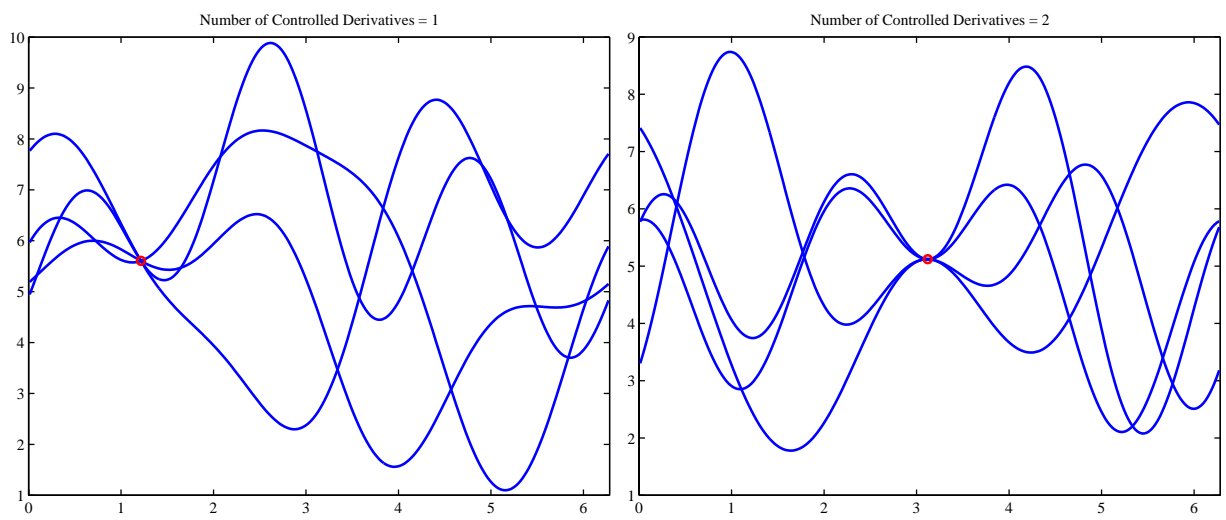

(a) (b)

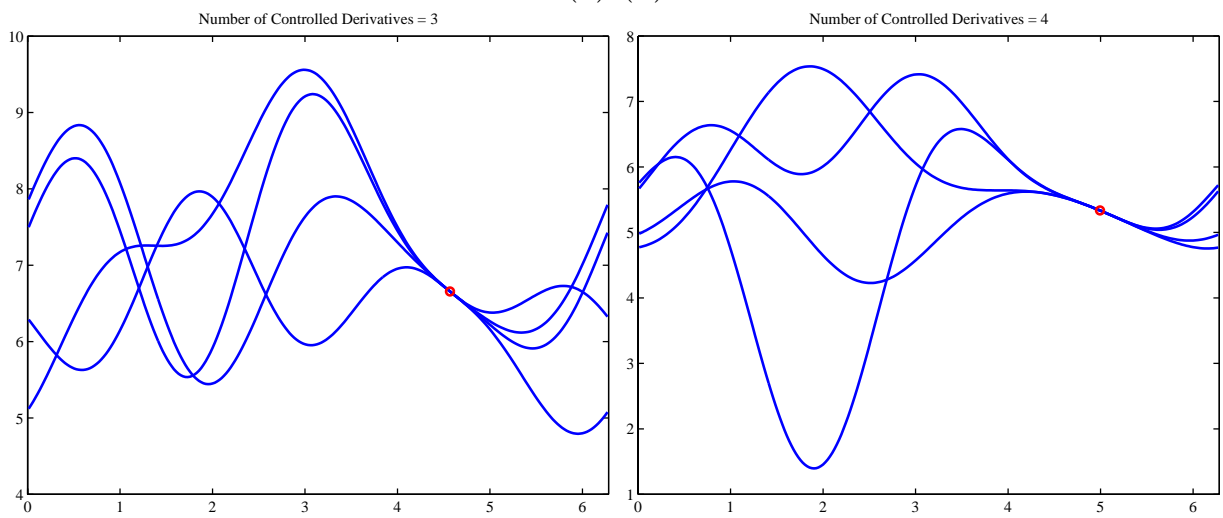

(c) (d)

FIG. 3. Families of random initial profiles $u(x, 0)(J=4)$. (a) $n=1$, (b) $n=2$, (c) $n=3$, (d) $n=4$ controlled initial derivatives. To avoid confusion notice that control of $n=1$ derivatives means that only $u\left(x_{0}, 0\right)$ is identical between the runs, $n=2$ means that $u\left(x_{0}, 0\right)$ and $u_{x}\left(x_{0}, 0\right)$ are identical, and so on.

slow governing cumulants. This separation of time scales, which fundamentally underlies the existence of a deterministic coarse equation closing with the lower cumulants, is discussed in more detail in [1].

As a sanity check, this algorithm is applied to a traditional continuum PDE timestepper "black box" first. Figures 4(a) and 4(b) show the results of applying our decision scheme to forward Euler finite-difference PDE solvers of the Burgers and $\mathrm{KdV}$ equations, respectively. A spatial mesh of $\Delta x=2 \pi / 100$ is adopted, and we define

$$
\begin{aligned}
u_{x}^{m k} & \equiv \frac{u_{(m+1) \Delta x, k h}-u_{(m-1) \Delta x, k h}}{2 \Delta x}, \\
u_{x x}^{m k} & \equiv \frac{u_{(m+1) \Delta x, k h}+u_{(m-1) \Delta x, k h}-2 u_{m \Delta x, k h}}{\Delta x^{2}}, \\
u_{x x x}^{m k} & \equiv \frac{u_{x}^{m+1, k}+u_{x}^{m-1, k}-2 u_{x}^{m k}}{\Delta x^{2}} \\
u_{\mathrm{avg}}^{m k} & \equiv \frac{u_{(m+1) \Delta x, k h}+u_{m \Delta x, k h}+u_{(m-1) \Delta x, k h}}{3} .
\end{aligned}
$$



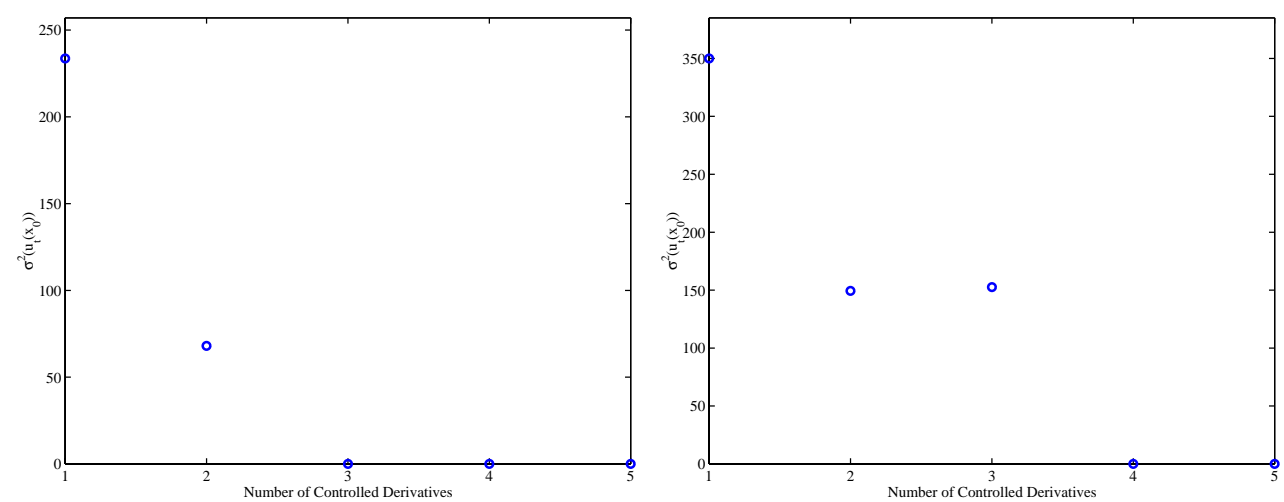

(a) (b)

FIG. 4. (a) Identification of the order of the highest (spatial) derivative in the Burgers finitedifference PDE time-stepper (3.9). (b) Identification of the order of the highest (spatial) derivative for the KdV finite-difference PDE time-stepper (3.10).

We use

$$
\frac{u_{m \Delta x,(k+1) h}-u_{m \Delta x, k h}}{h}=\nu u_{x x}^{m k}-u_{m \Delta x, k h} u_{x}^{m k}
$$

to integrate the Burgers equation forward and

$$
\frac{u_{m \Delta x,(k+1) h}-u_{m \Delta x, k h}}{h}=6 u_{\mathrm{avg}}^{m k} u_{x}^{m k}-u_{x x x}^{m k}
$$

to integrate the $\mathrm{KdV}$ equation forward. $u\left(x_{0}, k h\right)$ is obtained by cubic splines over $\left\{u_{m \Delta x, k h}\right\}$, and $u_{t}\left(x_{0}, 0\right)$ is evaluated by finite differences, the same as in (3.3). As can be seen in Figures 4(a) and 4(b), $N$ is identified to be 2 using the Burgers PDE time-stepper and 3 using the KdV PDE time-stepper: the variances drop by more than four decades in both cases when going from $N$ to $N+1$ controlled derivatives. To see where the remaining "noise" comes from, note that

$$
u\left(x_{0}, \Delta\right)-u\left(x_{0}, 0\right)=u_{t}\left(x_{0}, 0\right) \Delta+u_{t t}\left(x_{0}, 0\right) \frac{\Delta^{2}}{2}+\cdots,
$$

and clearly $u_{t t}(x, 0)$ has higher-than- $u_{x}^{(N)}$ spatial derivative dependencies, which are, however, scaled by $\Delta$ compared to the leading term. Thus the sample variances should drop by $\sim \Delta^{2}$ for $n>N$, which explains the observed magnitude of the four-decade decrease.

We then apply the identification scheme to the microscopic simulator of section 2, with the lifting and restriction operators constructed in Appendix A. The results are shown in Figure 5. Under favorable conditions such as $\nu=1$ and $m=100$, it takes about 10 minutes of computer time on a single $1 \mathrm{GHz}-\mathrm{CPU}$ personal computer to obtain a reasonably good microscopic noise reduction so that the variance drops by about two decades going from $n=2$ to $n=3$. Under unfavorable conditions such as $\nu=0.1$ and $m=1$, it can take up to 1,000 minutes of computer time to obtain the same two-decade drop. Compared to the deterministic finite-difference PDE time-steppers, identification of a microscopic simulator is undoubtedly much more computationally intensive, even though fundamentally there is no difference between the two black boxes. The problem of microscopic noise reduction is a persistent 


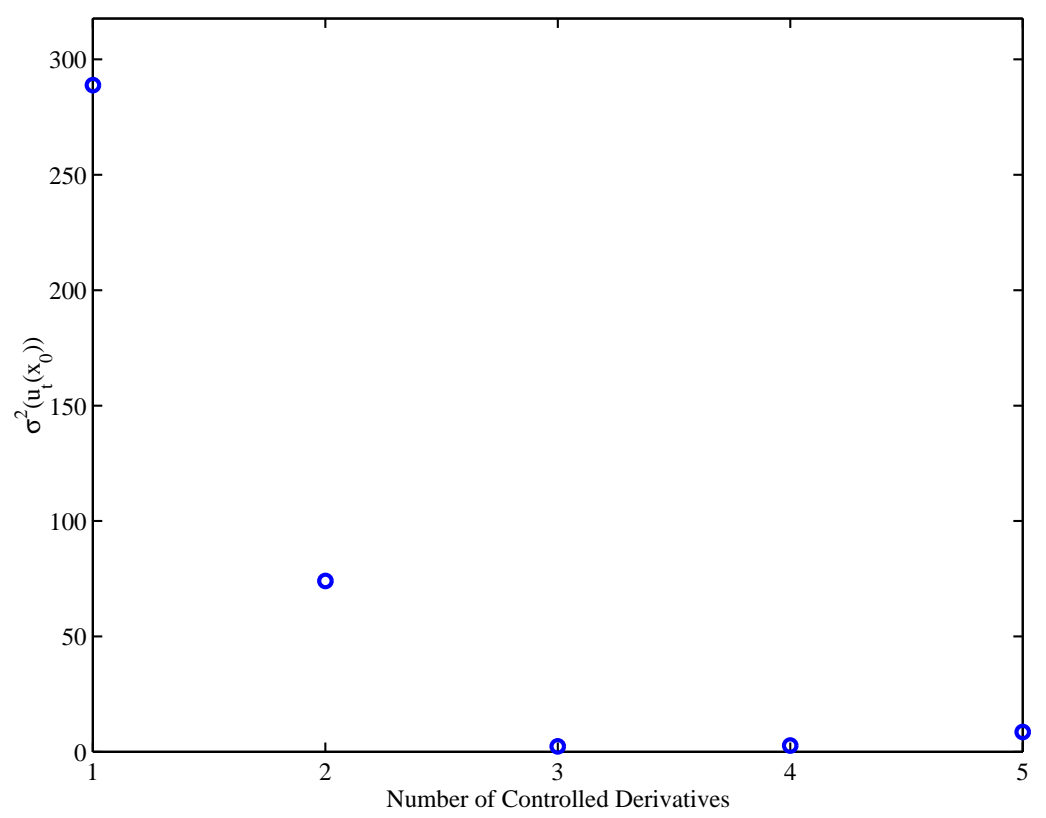

FIG. 5. Identification of the Burgers microscopic simulator of section 2 with the lifting and restriction operators of Appendix A. Here, $\nu=1, Z=10000, m=100, I=10, \Delta=0.01$.

issue among all equation-free methods including bifurcation [1], projective/gaptooth integration $[9,10]$, and identification, and calls for a unified treatment.

Here, one must pay special attention to the rank $(M)$ of the restriction operator (see Appendix A). As can be seen in Figures 1 and 2, our proposed restriction operator satisfies the constraint of reversibility and also accurately represents the profile's long-time evolution. However, these merits do not guarantee automatically good short-time $\tilde{u}_{t}$ estimates by finite difference. Special attention must be paid to the restriction operator $\hat{\mathcal{M}}$ : for example, if the highest harmonic in $(3.2)$ for $u(x, 0)$ is $L$, then with $M=L$ we can get a good reversibility test of $u(x, 0)$. Unless we use $M=2 L$ for restricting the Burgers microscopic dynamics, however, we would not get a good estimate of $u_{t}$, because the nonlinear interaction $u u_{x}$ in (2.1) creates higher harmonics in $u_{t}$ up to $2 L$. If $M=L$ is still used, it is equivalent to forcing a least-square projection of a $4 L+1$ vector to a $2 L+1$ subspace, which may work well enough in the long term but is too inaccurate for short-term finite-difference estimates. Unless this point is taken care of, the $u_{t}$ estimate using our $\hat{\mathcal{M}}$ is found to not even be superior to a crude bin-count density estimator with bin-width $(2 \pi / n) / 8$ about $x_{0}$, as $2 \pi / n$ is the shortest wavelength in $u(x, 0)$.

Last, we note that (3.1) represents a wide category of coarse dynamics; those with higher time-derivatives and mixed derivatives can be converted to a multivariate version of (3.1), and the identification scheme will still, in principle, work. A notable exception is the incompressible fluid case, where the sound-speed is infinite and the pressure plays the role of a global Lagrange multiplier. The incompressible fluid model is but a mathematical idealization of a certain physical limit. It is nonetheless useful and important enough; the fact that it is not directly amenable to our identification is worth mentioning. In general, the identification scheme presented here will not work 
for dynamics with instantaneous remote-action over macroscopic lengthscales such as

$$
u_{t}(x, t)=\int d \xi u(\xi, t) K(x-\xi),
$$

for which it is easy to show that $u_{t}(x, t)$ correlates with infinite number of local spatial derivatives $\left\{u_{x}^{(n)}(x, t)\right\}$.

4. Identifying conservation laws. In section 3 we addressed the concern of how to identify the highest spatial derivative of an unavailable coarse equation of the type (3.1). It is natural to try to decide other qualitative questions, for example whether the coarse dynamics conserve a specific quantity,

$$
G \equiv \int g\left(u, u_{x}, u_{x x}, \ldots, u_{x}^{\left(N^{\prime \prime}\right)}\right) d x
$$

or not. In the simplest case, we ask whether $g \equiv u$ is conserved. We note that it is equivalent to asking whether the right-hand side of (3.1) can be written as

$$
f\left(u, u_{x}, u_{x x}, \ldots, u_{x}^{(N)}\right)=-\partial_{x} j\left(u, u_{x}, u_{x x}, \ldots, u_{x}^{\left(N^{\prime}\right)}\right)
$$

or not. Alternatively, we ask whether there exists $j\left(u, u_{x}, u_{x x}, \ldots, u_{x}^{\left(N^{\prime}\right)}\right)$ such that

$$
\frac{d}{d t} \int_{x_{0}}^{x_{1}} u(x, t) d x=j\left(x_{0}, t\right)-j\left(x_{1}, t\right)
$$

for arbitrary $x_{0}, x_{1}$. Whereas in section 3 we try to identify features of $f\left(u, u_{x}, u_{x x}, \ldots\right.$, $\left.u_{x}^{(N)}\right)$ through (3.1), here we can try to identify consequences of $j\left(u, u_{x}, u_{x x}, \ldots, u_{x}^{\left(N^{\prime}\right)}\right)$ and its features through (4.3). The process of the baby-bathwater identification can be carried over; the only difference is that it is going to be a boundary scheme. In one dimension, the boundary sheme reduces to a two-point scheme as follows:

(i) Take an integer $n$, starting from 1.

(ii) Pick two random points $x_{0}$ and $x_{1}$ in the spatially periodic box.

(iii) Generate $2 n$ random numbers, which are to be designated $u\left(x_{0}, 0\right), u_{x}\left(x_{0}, 0\right)$, $u_{x x}\left(x_{0}, 0\right), \ldots, u_{x}^{(n-1)}\left(x_{0}, 0\right)$ and $u\left(x_{1}, 0\right), \quad u_{x}\left(x_{1}, 0\right), \quad u_{x x}\left(x_{1}, 0\right), \ldots$, $u_{x}^{(n-1)}\left(x_{1}, 0\right)$ of $u(x, 0)$.

(iv) Generate a conditionally random profile $u(x, 0)$ compatible with the $\mathrm{PBC}$ that is consistent with the above $u\left(x_{0}, 0\right), u_{x}\left(x_{0}, 0\right), u_{x x}\left(x_{0}, 0\right), \ldots, u_{x}^{(n-1)}\left(x_{0}, 0\right)$, and $u\left(x_{1}, 0\right), u_{x}\left(x_{1}, 0\right), u_{x x}\left(x_{1}, 0\right), \ldots, u_{x}^{(n-1)}\left(x_{1}, 0\right)$ requirements. This can always be done by (3.2) with $L>n$. As we discussed above, we have $2 L+1$ coefficients, even though there are $2 n$ constraints to satisfy, and we still have some random degrees of freedom left in $u(x, 0)$.

(v) Lift $u(x, 0)$ of $(3.2)$, run it in the microscopic simulator for time $\Delta$, restrict it back to $\tilde{u}(x, \Delta)$, and estimate:

$$
\tilde{U}_{t}(0) \equiv \frac{\int_{x_{0}}^{x_{1}} \tilde{u}\left(x^{\prime}, \Delta\right) d x^{\prime}-\int_{x_{0}}^{x_{1}} \tilde{u}\left(x^{\prime}, 0\right) d x^{\prime}}{\Delta} .
$$

(vi) Repeat step (v) $I$ times to obtain an ensemble-averaged $\tilde{U}_{t}(0)$ to reduce the microscopic noise. 

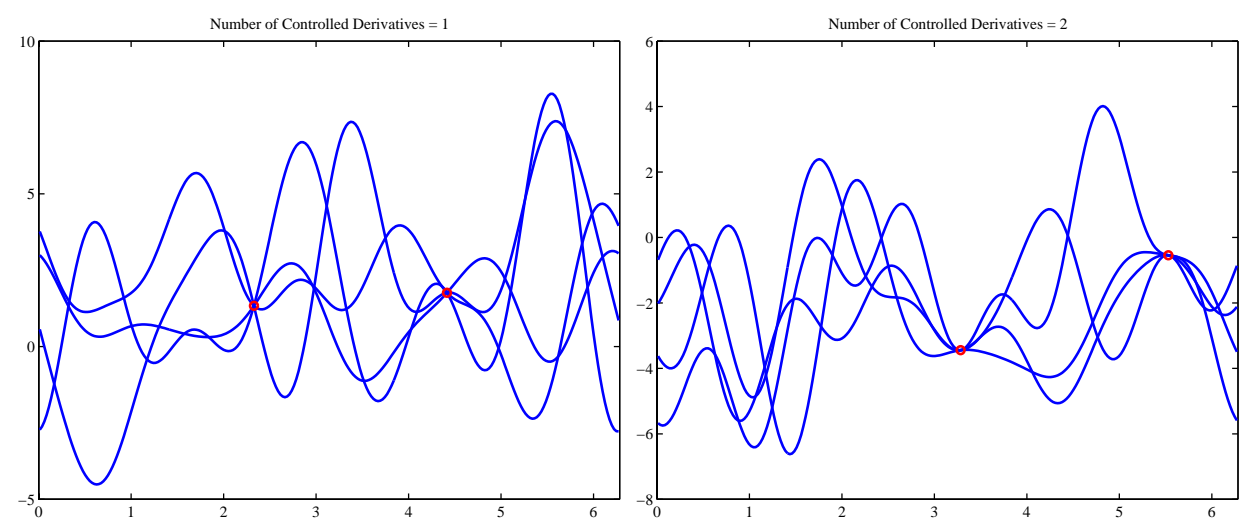

(a) (b)
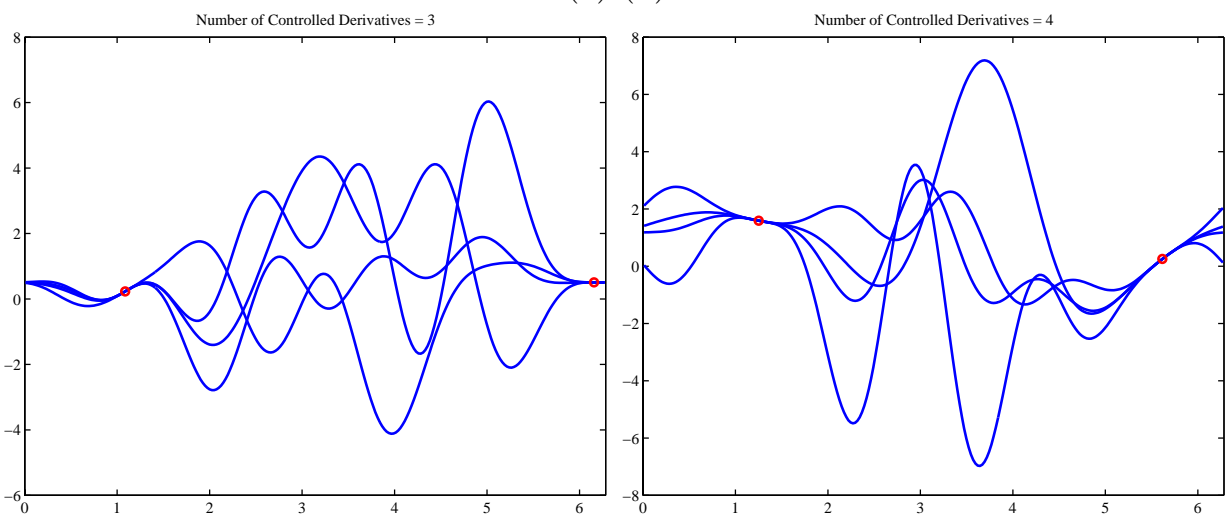

(c) (d)

FIG. 6. Families of random initial profiles $u(x, 0)(J=4)$ for conservation law identification. (a) $n=1$, (b) $n=2$, (c) $n=3$, (d) $n=4$ controlled initial derivatives.

(vii) Repeat step (iv) $J$ times; collect the $\tilde{U}_{t}(0)$ estimates:

$$
\left(\tilde{U}_{t}^{1}(0), \tilde{U}_{t}^{2}(0), \ldots, \tilde{U}_{t}^{J}(0)\right) ;
$$

compute the sample variance $\sigma^{2}\left(\tilde{U}_{t}(0)\right)$.

(viii) Repeat step (ii) $K$ times; compute the averaged sample variance $\left\langle\sigma^{2}\left(\tilde{U}_{t}\right)\right\rangle_{n}$.

(ix) Go back to step (i); $n \rightarrow n+1$. A conservation law is positively identified when going from $n=N^{\prime}$ to $n=N^{\prime}+1$ the averaged sample variance $\left\langle\sigma^{2}\left(\tilde{U}_{t}\right)\right\rangle_{N^{\prime}+1}$ decreases drastically to practically 0 .

Figure 6 plots families of initial profiles thus constructed with progressively more controlled initial derivatives. Figures 7(a) and 7(b) show the results of applying the identification scheme to the Burgers finite-difference PDE time-stepper (3.9) and the KdV finite-difference PDE time-stepper (3.10), respectively. $N^{\prime}$ is identified to be 1 for (3.9) and 2 for (3.10).

Two comments are in order: first, we probe the consequence of conservation (i.e., that boundary fluxes are the only cause of change for the conserved quantity in a domain); second, we obtain the highest spatial derivative of the conserved quantity $u$ in the constitutive equation for the flux. It is important to note that if the procedure progressively returns negative answers (e.g., if the sample variance is nonzero for a 

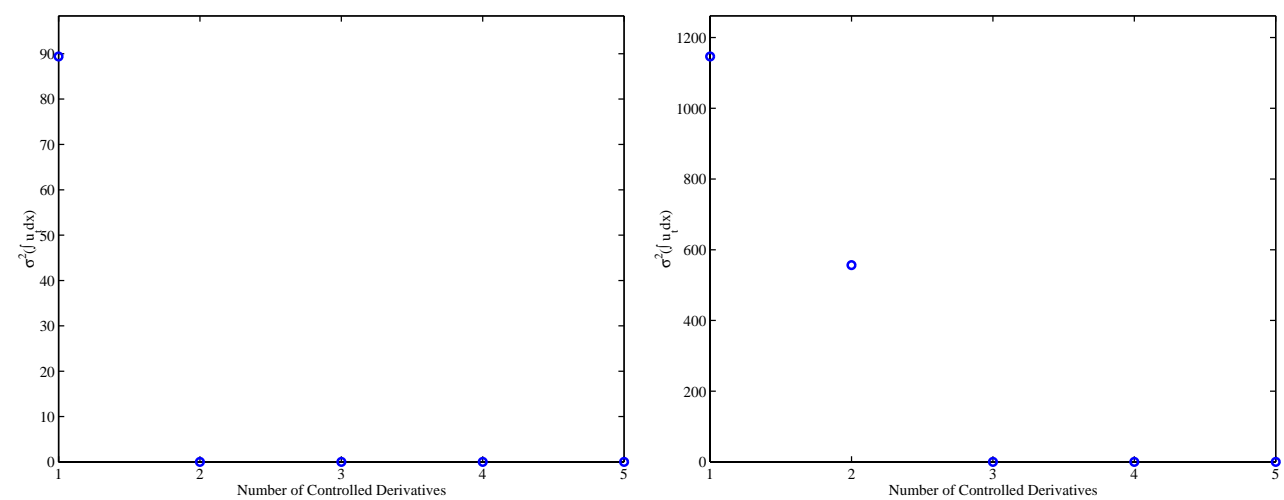

(a) (b)

FiG. 7. (a) Conservation of the Burgers finite-difference PDE time-stepper (3.9). (b) Conservation of the KdV finite-difference PDE time-stepper (3.10).

given number $n$ of controlled derivatives), this does not imply that a conservation law does not exist. It implies only that a conservation law with spatial derivatives up to the tested order $n$ does not exist. So it is sufficient but not necessary confirmation.

Note that $N^{\prime}$ is one order less than $N$ identified in section 3 in both cases. This is true in one dimension because of (4.2). So in one dimension, the baby-bathwater schemes give a definite answer to whether $u$ is conserved or not in finite $N-1$ steps, as long as $N$ is identified first.

5. Discussion. In section 4 we proposed methods to check whether a coarse quantity (such as the mass corresponding to the coarse density $g\left(u, u_{x}, u_{x x}, \ldots, u_{x}^{\left(N^{\prime \prime}\right)}\right)$ ) is conserved, without knowledge of the coarse evolution equation. The obvious question that arises is, How do we know which $g$ to check? The path that we suggest here, in the equation-free setting, is to examine the consequences of conservation laws. For example, consider the conservation of (linear) momentum. An equivalent statement, through Noether's theorem [21], is the existence of translational invariance. If we numerically establish the latter, then we can claim the former. Let us then consider initial conditions to the available integrator which are shifts of an original profile (e.g., $u\left(x-x_{0}\right), u\left(x-\left(x_{0}+\epsilon\right)\right), u\left(x-\left(x_{0}+2 \epsilon\right)\right)$, etc.). Then if we time evolve the problem, using our microscopic time-stepper, and the equation is translationally invariant, upon reaching the integration reporting horizon we can backshift the profile (by the original shift amount). If all back-shifts provide an identical profile, we can conclude translational invariance and hence linear momentum conservation. An additional note of caution is that the examination of such consequences is relevant when Noether's theorem applies and hence when there is an underlying Lagrangian/Hamiltonian structure in the problem (we discuss separately the issue of Hamiltonian nature below). Notice, however, that modulo the proviso of "Hamiltonianity"; this methodology can be used to establish additional dynamical invariants; e.g., the invariance with respect to phase of the evolution of a field can be related to norm invariance, etc.

Testing for an underlying Hamiltonian structure can proceed in a similar fashion through its correlation with invariance with respect to time reversal. The crudest way to examine this is by simply running the integrator with a negative time-step (if that option is available). A more refined way to check the same symmetry is by examining computations of the spectrum (e.g., eigenvalues) of linearization of the coarse PDE. 
In particular, a straightforward consequence of the Hamiltonian nature is that all linearization eigenvalues should come in quartets; namely, if $\lambda$ is an eigenvalue, then so are $-\lambda, \lambda^{\star},-\lambda^{\star}$, where ${ }^{\star}$ denotes complex conjugation. It is fortunate that timestepper based numerical analysis techniques for the numerical approximation of the leading spectrum of such a linearization are well developed for the case of large scale continuum simulations (see, for example, $[22,23,24,25,26,27]$ ). If an eigenvalue $\lambda$ of the linearization is identified, matrix-free eigencomputations with shift can be used to explore the existence of the $-\lambda$ eigenvalue (in general, real eigenvalues will come in pairs and complex conjugate eigenvalues in quartets).

While coarse time reversibility can be tested by exploring the spectrum of the linearization, it raises the interesting question of how to integrate backward in time with the microscopic code. If the coarse dynamic behavior is slow (i.e., under appropriate spectral gap conditions for the coarse equation), we can use short bursts of forward in time microscopic simulation to estimate the time derivative of the coarse variables. We can then use this (regularized through forward integration) estimate to take a backward projective integration step. The procedure is then repeated: short forward integration, coarse time-derivative estimation, and a new backward projective step. This "see-saw" forward-backward coarse integration procedure can also be used on stiff systems of ODEs and even dissipative PDEs under the appropriate conditions to evolve trajectories backwards on a slow manifold. The numerical analysis of these algorithms in the continuum case is an interesting subject in itself, and we are currently pursuing it [28]. It is interesting that the technique, in the molecular dynamics case, can be used to coarsely integrate backward in time on a free energy surface and thus help molecular simulations escape from free energy minima; we have already confirmed this in the case of Alanine dipeptide folding in water at room temperature through molecular dynamics simulations [29].

Finally, a more complicated question than checking the existence of one (a specific, and hence related to a specific invariance, in accordance with the above discussion) integral of the motion is the one of integrability. The latter necessitates infinite integrals of the motion, normally established by means of identifying Lax pairs and using the inverse scattering transformation machinery [30]. However, one can also use in this case consequences of integrability to establish it. For instance, in recent work [31] it was qualitatively argued (and verified through numerical experiments in different settings) that a feature particular to integrable Hamiltonian systems is the presence of double continuous spectrum eigenvalues when linearizing around a (coarse PDE) solitary wave under PBCs. These as well as other criteria (such as the existence of point spectrum eigenvalues in the spectral gap [32]) can also be (conversely) used to potentially rule out the existence of integrable structure. In short, the spectral properties of the coarse PDE linearization can be used to establish or disprove not only the Hamiltonian (see above) but also potentially the integrable nature of the flow. While these are just initial thoughts towards attempting to decide vital questions about the nature of the unavailable closed equation, it is important to note that what is computationally involved is a time-stepper-based identification of facts about the spectrum of the linearization of an operator. This "computational technology" is quite mainstream in the case of large scale continuum simulators and can be straightfowardly adapted to the case of coarse time-steppers in conjunction with the lifting-restriction steps. Variance reduction will clearly be the most significant step in the wide applicability of these and similar-spirited approaches. 
Appendix A. Coarse density lifting/restriction operators. For a coarse field $\mathbf{u}$, the lifting operator $\hat{\mu}$ generates a microstate $\mathbf{U}: \mathbf{U}=\hat{\mu} \mathbf{u}$. Similarly, for a microstate $\mathbf{U}$, the restriction operator $\hat{\mathcal{M}}$ returns a coarse field estimate $\tilde{\mathbf{u}}: \tilde{\mathbf{u}}=\hat{\mathcal{M}} \mathbf{U}$. Both $\hat{\mu}$ and $\hat{\mathcal{M}}$ can be one-to-one or one-to-many operators, but we demand that $\hat{\mathcal{M}} \hat{\mu} \rightarrow \hat{I}$ asymptotically when the wavelength of $\mathbf{u}$ is large enough compared to the microscopic length scale [6]. For one-dimensional coarse density field $u(x)$ under $x \in[0,2 \pi)$ PBCs, we use the following $\hat{\mu}, \hat{\mathcal{M}}$ operators for the sake of definiteness in numerical experiments, even though their construction is not unique.

The lifting operator $\hat{\mu}, u(x) \rightarrow\left\{x_{i}\right\}$ :

(i) Estimate $u_{\max }^{\text {approx }} \approx u_{\max } \equiv \max _{x \in[0,2 \pi)} u(x)$. Pick $u_{\text {safe }}$ that is "safely" greater than $u_{\max }$; for example, $u_{\mathrm{safe}}=1.1 u_{\max }^{\mathrm{approx}}$.

(ii) Define $N^{\prime} \equiv\left\lceil 2 \pi u_{\text {safe }} Z\right\rceil$. Create $N^{\prime}$ particles $\left\{x_{i}\right\}$ with each $x_{i}$ independently drawn from uniform distribution on $[0,2 \pi)$.

(iii) Go to each particle $i$; randomly decimate it with probability $1-\frac{2 u\left(x_{i}\right)}{u_{\max }^{\text {appox }}+u_{\text {safe }}}$. Count the total number of surviving particles $N^{\prime \prime}$.

(iv) Compute quadrature,

$$
Q \equiv Z \int_{0}^{2 \pi} u(x) d x
$$

randomly round to $N=\lceil Q\rceil$ or $N=\lceil Q\rceil+1$ such that $\langle N\rangle=Q$. Randomly pick $N^{\prime \prime}-N$ particles out of the $N^{\prime \prime}$ survivors and decimate them. We now have a set of particles $\left\{x_{i}\right\}$, totally numbered either $\lceil Q\rceil$ or $\lceil Q\rceil+1$.

The restriction operator $\hat{\mathcal{M}},\left\{x_{i}\right\} \rightarrow \tilde{u}(x)$ :

(i) Define a microscopic density function,

$$
a(x) \equiv \frac{1}{Z} \sum_{i=1}^{N} \delta\left(x-x_{i}^{-}\right),
$$

and a corresponding cumulant function,

$$
c(x) \equiv \int_{0}^{x} a\left(x^{\prime}\right) d x^{\prime} .
$$

Clearly, at the the first, second, and third particle positions $x_{n_{1}}, x_{n_{2}}, x_{n_{3}}$, $c\left(x_{n_{1}}\right)=1 / Z, c\left(x_{n_{2}}\right)=2 / Z, c\left(x_{n_{3}}\right)=3 / Z$, etc. And we have $c(0)=0$, $c(2 \pi)=N / Z$.

(ii) Define a residual function $r(x)$,

$$
r(x) \equiv c(x)-\frac{N x}{2 \pi Z},
$$

which is the difference between $c(x)$ and the cumulant of a homogenized particle gas background. The idea is that $r(0)=r(2 \pi)=0$, so it is a periodic function and can be approximated by

$$
r(x) \approx \tilde{r}(x)=\sum_{n=1}^{M} a_{n}(\cos (n x)-1)+b_{n} \sin (n x) .
$$

In fact, a sound strategy is to least-square fit $\tilde{r}(x)$ (its $\left\{a_{n}\right\},\left\{b_{n}\right\}$ coefficients) to $r(x)$ at $x=x_{n_{i}}$ 's, where $\left\{x_{n_{i}}\right\}$ is the sorted list of $\left\{x_{i}\right\} . r(x)$ can be easily evaluated at $x_{n_{i}}$ 's, noting the last sentence of step 1 . 
(iii) The coarse density estimate can be obtained by taking the derivative of $\tilde{c}(x) \equiv$ $N x / 2 \pi Z+\tilde{r}(x)$

$$
\tilde{u}(x)=\frac{N}{2 \pi Z}+\sum_{n=1}^{M}-n a_{n} \sin (n x)+n b_{n} \cos (n x) .
$$

It is worth noting that although the constructed $\hat{\mathcal{M}}$ depends on $M$, it satisfies the particle number conservation exactly because the finite harmonics all integrate to zero, and only the background contribution remains. In fact, $\langle\hat{\mathcal{M}} \hat{\mu}\rangle$ also satisfies exact particle number conservation to the original $u(x)$ under probabilistic average. Further, one can show $\langle\hat{\mathcal{M}} \hat{\mu}\rangle=\hat{I}$ exactly for $u(x)$ in the first $M$ harmonics subspace.

Acknowledgment. The authors would like to thank Frank Alexander for stimulating discussions.

\section{REFERENCES}

[1] I.G. Kevrekidis, C.W. Gear, J.M. Hyman, P.G. Kevrekidis, O. Runborg, and C. Theodoropoulos, Equation-free multiscale computation: Enabling microscopic simulators to perform system-level tasks, Communications in the Mathematical Sciences, submitted.

[2] A.G. Makeev, D. Maroudas, and I.G. Kevrekidis, Coarse stability and bifurcation analysis using stochastic simulators: Kinetic Monte Carlo examples, J. Chem. Phys., 116 (2002), pp. 10083-10091.

[3] A.G. Makeev, D. Maroudas, A.Z. Panagiotopoulos, And I.G. Kevrekidis, Coarse bifurcation analysis of kinetic Monte Carlo simulations: A lattice-gas model with lateral interactions, J. Chem. Phys., 117 (2002), pp. 8229-8240.

[4] C.W. Gear And I.G. KevreKidis, Projective methods for stiff differential equations: Problems with gaps in their eigenvalue spectrum, SIAM J. Sci. Comput., 24 (2003), pp. 1091-1106.

[5] G.M. Shroff And H.B. Keller, Stabilization of unstable procedures: The recursive projection method, SIAM J. Numer. Anal., 30 (1993), pp. 1099-1120.

[6] C.W. Gear, I.G. Kevrekidis, and C. Theodoropoulos, Coarse integration/bifurcation analysis via microscopic simulators: Micro-Galerkin methods, Computers and Chemical Engineering, 26 (2002), pp. 941-963.

[7] J. Li, K.J. Van Vliet, T. Zhu, S. Yip, And S. Suresh, Atomistic mechanisms governing elastic limit and incipient plasticity in crystals, Nature, 418 (2002), pp. 307-310.

[8] K.J. Van Vliet, J. Li, T. Zhu, S. YiP, And S. Suresh, Quantifying the early stages of plasticity through nanoscale experiments and simulations, Phys. Rev. B, 67 (2003), 104105.

[9] I.G. Kevrekidis, Coarse Bifurcation Studies of Alternative Microscopic/Hybrid Simulators, Plenary Lecture, CAST Division, AIChE Annual Meeting, Los Angeles, 2000. Slides can be obtained at http://arnold.princeton.edu/ yannis/.

[10] C.W. Gear, J. Li, AND I.G. KevreKidis, The gap-tooth method in particle simulations, Phys. Lett. A, to appear.

[11] W. E And B. Engquist, The heterogeneous multi-scale methods, Communications in Mathematical Sciences, 1 (2003), pp. 87-132.

[12] J.D. ColE, On a quasilinear parabolic equation occurring in aerodynamics, Quart. Appl. Math., 9 (1951), pp. 225-236.

[13] E. Hopf, The partial differential equation $u_{t}+u u_{x}=\mu u_{x x}$, Comm. Pure Appl. Math., 3 (1950), pp. 201-230.

[14] S. RoBerTs, Convergence of a random walk method for the Burgers equation, Math. Comp., 52 (1989), pp. 647-673.

[15] J. Li, C.W. Gear, And I.G. Kevrekidis, Particle solution of the Burgers equation and microscopic correlations, to be submitted.

[16] J. Li, D. LiaO, AND S. YIP, Nearly exact solution for coupled continuum/MD fluid simulation, J. Comput.-Aided Mater. Des., 6 (1999), pp. 95-102.

[17] J. Li, D. LIAO, AND S. YIP, Coupling continuum to molecular-dynamics simulation: Reflecting particle method and the field estimator, Phys. Rev. E, 57 (1998), pp. 7259-7267.

[18] L.B. LuCY, A numerical approach to the testing of the fission hypothesis, Astronom. J., 82 (1977), pp. 1013-1024. 
[19] A. Chertock And D. Levy, Particle methods for dispersive equations, J. Comput. Phys., 171 (2001), pp. 708-730.

[20] A. Chertock and D. Levy, A particle method for the KdV equation, J. Sci. Comput., 17 (2002), pp. 491-499.

[21] V.I. Arnold, Mathematical Methods of Classical Mechanics, Springer-Verlag, New York, 1989.

[22] K.N. Christodoulou ANd L.E. Scriven, Finding leading modes of a viscous free surface flow: An asymmetric generalized eigenproblem, J. Sci. Comput., 3 (1988), pp. 355-406.

[23] I. Goldhirsch, S.A. Orszag, and B.K. Maulik, An efficient method for computing leading eigenvalues and eigenvectors of large asymmetric matrices, J. Sci. Comput., 2 (1987), pp. $33-58$.

[24] Z. Bai, J. Demmel, J. Dongarra, A. Ruhe, and H. van der Vorst, eds., Templates for the Solution of Algebraic Eigenvalue Problems: A Practical Guide, Software Environ. Tools 11, SIAM, Philadelphia, 2000.

[25] W.E. ARNoldi, The principle of minimized iterations in the solution of the matrix eigenvalue problem, Quart. Appl. Math., 9 (1951), pp. 17-29.

[26] C. LANCzos, An iteration method for the solution of the eigenvalue problem of linear differential and integral operators, J. Research Nat. Bur. Standards, 45 (1950), pp. 255-282.

[27] L.S. Tuckerman AND D. BARKLey, Bifurcation analysis for time-steppers, in Numerical Methods for Bifurcation Problems and Large-Scale Dynamical Systems, IMA Vol. Math. Appl. 119, E. Doedel and L. S. Tuckerman, eds., Springer, New York, 2000, pp. 453-466.

[28] C.W. Gear and I.G. Kevrekidis, Computing in the past with forward integration, Phys. Lett. A, submitted.

[29] G. Hummer and I.G. Kevrekidis, Coarse molecular dynamics of a peptide fragment: Free energy, kinetics and long-time dynamics computations, J. Chem. Phys., 118 (2003), pp. 10762-10773.

[30] M.J. Ablowitz And H. Segur, Solitons and the Inverse Scattering Transform, SIAM Stud. Appl. Math. 4, SIAM, Philadelphia, 1981.

[31] P.G. Kevrekidis And N.R. Quintero, Using the Continuous Spectrum to Feel Integrability, the Effect of Boundary Conditions, preprint.

[32] P.G. Kevrekidis, Integrability revisited: A necessary condition, Phys. Lett. A, 285 (2001), pp. 383-389. 